\title{
Specification of Secure Distributed Collaboration Systems
}

\author{
Anand R. Tripathi, Tanvir Ahmed, and Richa Kumar \\ \{tripathi, tahmed, richa\}@cs.umn.edu \\ Department of Computer Science \\ University of Minnesota, Minneapolis MN 55455
}

\begin{abstract}
The focus of this paper is on a specification model for defining security and coordination policies for distributed collaboration and workflow systems. This work is motivated by the objective to build distributed collaboration systems from their high level specifications. We identify here unique requirements for secure collaboration, specifically role admission and activation constraints, separation of duties, dynamic access control, and a model for multi-user participation in a role. We present a role-based model for specifying coordination and dynamic security requirements in collaboration systems. It also supports hierarchical structuring of a large collaboration environment using the concept of activities, which define a naming scope and a protection domain to specify security and coordination policies. We have implemented this specification model in XML and used it to construct the runtime environments for distributed collaboration systems using a policy based middleware.

Keywords: Distributed collaboration, Role based access control, Security policy specification, Decentralized management of collaboration systems.
\end{abstract}

\section{Introduction}

The objective of our research is to realize distributed CSCW (Computer Supported Cooperative Work) systems from their high level specifi cations. In this paper, our primary focus is on the specifi cation model for secure distributed 
collaboration systems. The specifi cation model is developed to address security and coordination requirements of these systems. The approach to realize the runtime environment from this specifi cation model by means of a generic policydriven middleware is presented in [23]. A policy-based approach decouples the coordination and security aspects of a collaboration system from the implementation of the collaboration objects. This makes it easy for different policies to be plugged in, allowing flexibility in designing as well as modifying a collaboration environment. Several factors motivate this approach. Collaboration environments often span multiple administrative domains. The requirements of a collaboration system may evolve with changes in administrative policies and user experience. With advances in technology, new devices, tools or artifacts may need to be integrated into a collaboration environment. This can warrant a reconfi guration of the system with changes in the governing policies.

Coordination requirements in collaboration systems include synchronization and precedence constraints. The general requirements of security in such systems are related to confi dentiality and integrity of the shared data, privacy of a user's actions, auditability and non-repudiation of users' actions, and the integrity of operations to ensure that only authorized participants perform certain sensitive tasks. Additionally, CSCW systems pose several unique security requirements not present in traditional operating systems and databases [8]. An important characteristic of collaboration environments is the need to have dynamic security policies such as dynamic "separation of duties" constraints as well as context sensitive access control mechanisms that depend on participants' past actions and the execution state of the collaboration. The existing research on security in collaboration targets either synchronous groupware applications or asynchronous workflow. However, the security requirements and solutions proposed for these two areas are different, when, in practice, synchronous collaboration comes into existence in many stages of asynchronous workflow. Shen and Dewan [4] presented an access control model with a set of rights that are unique to shared-view based GUI interface control in groupware environments. In workflow, database oriented security is prevalent $[1,10]$.

In this paper, we present a specifi cation model that can capture the various coordination and security requirements outlined above. Past research in this area, such as Task Based Access Control (TBAC) [19, 1], shows that the specifi cation of many dynamic security requirements necessitates a unifi ed model for expressing coordination and security policies. Our objective is similar to COCA [12] and DCWPL [2] in their approach of constructing a distributed collaboration environment from a high level specifi cation. However, these specifi cations are limited to coordination policies. They do not provide adequate support for security policies like user-assignment to roles, dynamic access control or 
history based security constraints. Moreover, they do not provide any mechanisms for hierarchical organization of collaboration systems and context sensitive privileges.

The role based specifi cation model for collaboration systems presented here supports the expression of requirements such as "separation of duties", intra-role and inter-role coordination, admission control policies, role activation constraints, and dynamic access control policies. An important aspect of this work is reflected by the fact that the specifi cation model has been integrated with a policy-driven middleware to realize the runtime environments for collaboration systems [23]. In the specifi cation model, we introduce the notion of reusable activity templates, which provide a facility to dynamically create activity instances, which can be hierarchically nested. Based on this specifi cation model, we have devised an XML schema to express collaboration specifi cations using XML. In this paper, for the sake of simplicity and brevity, rather than using XML, we use a notation that is simple and conceptually easy to follow.

In the next section, we present our role based collaboration model. Section 3 discusses the requirements for secure collaboration systems. Our specifi cation model is described in Section 4. Section 5 shows how the specifi cation model is used by a policy based middleware to build runtime collaboration environments. Sections 6 and 7 present the related work and the conclusions.

\section{A Role Based Collaboration Model}

In a role based security model, a role represents a set of privileges [15]. One can view a role as the characterization of a protection domain. A user assigned to a role acquires those privileges. The use of role based security policies in collaboration and workflow systems has been found to be quite natural as participants perform a set of well-defi ned tasks pertaining to their expertise and responsibilities in the organization [3]. However, in traditional RBAC (Role Based Access Control) model [15], all the privileges in a role are assigned statically thus lacking support for dynamic access control policies. As roles are globally defi ned, context based access control is not supported, unless new roles are created based on the context. Additionally, the issue of users' assignment to roles is not addressed.

In an organization, many activities exist and new activities are constantly created. Existing roles and new roles participate in these activities. A large collaborative environment may sometimes need to be structured hierarchically. 
For example, a system supporting a team based design project running over several weeks or months may need to have several dynamically created synchronous shared 'whiteboard-like" activities of relatively shorter life-spans.

In workflow systems, such as $[1,10]$, dynamic task assignments to roles following authorization constraints are addressed. Integration of roles, object types, and time intervals in a task-based active security policy specifi cation is addressed in [10]. Though these works motivated us, they do not address policy requirements on task creation, metapolicies such as users assignment to roles, and administrative meta-policies such as who can enforce collaboration policies. Also, they lack a framework for realizing a system from its specifi cations.

In our collaboration model, an activity is an abstraction of a shared task. An activity defi nes how a group of users cooperate towards some common objectives by performing their individual tasks on a set of shared objects. It represents a protection domain and a scope for the roles, objects, and privileges in a collaboration. In an activity, users are represented by their roles, and roles within an activity are assigned privileges to perform certain tasks. We term these role specifi c tasks as operations. An operation can be method invocations on shared objects, synchronization actions, or activity management actions. Besides the application-defi ned roles in an activity, there are several administrative roles or meta-roles, such as Creator and Owner of an activity. An activity can be structured hierarchically, consisting of multiple nested concurrent activities.

An activity template specifi es a generic collaboration pattern among a set of roles using some shared objects. To support hierarchical structuring of a large collaboration system into smaller activities, an activity template specifi cation can defi ne nested activity templates. Activities are instantiated from templates. Any number of instances of a template can be dynamically and concurrently created.

In a collaboration environment, users may be distributed over a network, and they may be associated with different administrative domains. In a typical situation, a user participates in a collaboration by joining a role in an activity. A role operation can result in a sequence of interactions between the invoker and a set of shared objects. This represents a session in the context of that role operation. 


\section{Requirements for Secure Collaboration}

In this section, we identify several important security requirements that a role-based model for collaboration systems should support.

\subsection{Role Admission Constraints}

A role based model needs to address role admission constraints. Role admission constraints specify the conditions that need to be satisfi ed when a user requests to join a role. The admission constraints can be based on several different kinds of criteria. It may specify a list of users that should be allowed to join a role, or it may also include a list of those who should never be admitted. A role admission constraint can be based on previous qualifi cations, which could be that the requesting user is currently admitted in some other given roles. Additional role admission constraints can be based on cardinality, i.e. a count specifying the maximum number of participants allowed to be admitted in the role. Another kind of constraint could specify the events that must happen before a user could be admitted in a role.

\subsection{Coordination Requirements}

Coordination between participants in different roles within an activity is referred to as inter-role coordination. The primary motivation of this requirement is to enforce precedence constraints among different roles' operations. For example, an inter-role coordination requirement in a conference workflow can be that the Reviewer role can review a paper after the Author role of the paper has made the fi nal submission.

However, when multiple users are allowed to be present simultaneously in a role, users within the role may require to coordinate among themselves, which is be termed as intra-role coordination. When multiple users are present in a role, they can participate either independently or cooperatively. In independent participation, all of the role specific task responsibilities are assumed individually by a participant, irrespective of the presence of the other participants. For example, every participant of a conference Reviewer role has to independently write a review.

On the other hand, when the participants in a role are assuming task responsibilities cooperatively, they coordinate among themselves on deciding who is performing which role specific task. For example, in a hospital patient ward, several nurses may be present in the role of nurse-on-duty. However, some medical procedure on a patient may need 
to be performed only once by any of the members. Another type of cooperation may require a task to be performed by all the participants of a role, like jointly opening a bank vault. Moreover, in some collaboration environments there may be no coordination among participant actions, e.g. in an unrestricted whiteboard sharing.

\subsection{Separation of Duties}

Several researchers have discussed "separation of duties" requirements in role-based access control models [17, 16, 20], namely static separation of duty, dynamic separation of duty, user-user conflict, user-role conflict, object based separation of duty, and operational separation of duty. A specifi cation model for collaboration has to be able to express these constraints. The notion of static separation of duty requires that two given roles should never be assigned to the same person. The concept of dynamic separation of duty requires that two given roles cannot be concurrently assigned to or activated by the same person. The notion of user-user conflict requires that two particular users should not be assigned to the same role. A user-role conflict specifi es that a specifi ed user should never be assigned to a given role. Another kind of requirement is object based separation of duty. A user cannot perform multiple operations of the same object by participating in two different roles. The operational separation of duty requires that no single participant of a role can perform all the operations related to a business transaction.

\subsection{Dynamic Access Control Policies}

The privileges assigned to a user in a role may change with time due to the actions executed by other participants. Sometimes permissions may change due to the user's own actions, such as making a fi nal agreement on a document. In some situations, a role should be allowed to execute an operation only after another role has performed some other action. For example, in a course examination activity, a security requirement can be that students can only view the question after the examiner has released it and only during the specifi ed time period of the exam-session activity.

Several types of "separation of duty" constraints and history-based access control conditions also fall into the category of dynamic access control policies. In the context of role based access control, dynamic access control policies have to address issues, such as constraints requiring a maximum and minimum number of participants that must be present for a role to perform any operation.

Traditional RBAC, and most of the existing MAC (Mandatory Access Control) and DAC (Discretionary Access 
Control) style security policies are static, i.e., they do not depend on time or other events. Though permissions are assigned to roles, they may be specifi c based on various contexts and can be activated in those contexts. For example, in the course examination activity, a candidate can access the answer book only during an exam session. Additional context based access control may be related to physical environment's events. For example, in the course examination activity, context based access control may specify that the candidates can access answer books only when they are physically present in the class room and that too only during a predefi ned period.

\subsection{Privacy}

Privacy becomes an issue when one may need to hide the identity of one participant from another. In such cases, the presence of a participant may be only visible through his/her role or a pseudonym in a role but not by name. It may be required to hide identities of the participants of a role from other roles. Consider a course examination activity, which has two roles: the Candidate takes the exam and the Grader grades the answer book. A requirement can be that the graders do not know the identities of the Candidate role's participants. A similar collaboration requirement can specify that only the owner of a role knows the identities of the role participants. For example, in a conference submission workflow, the Program Chair role owns the Reviewer roles, who review submitted papers. However, none other than the users in the Program Chair role are permitted to view the identities of the participants of the Reviewer roles.

\subsection{Meta-level Security Policies}

An activity requires many administrative security policies: who can defi ne new activities or instantiate an activity; during the lifetime of an activity, who can change various policies and enforce additional constraints on shared objects. These meta level administrative policies need to be specifi ed based on meta roles, such as Creator and Owner for various entities like activities, roles, and objects. Policies need to be specifi ed on who can join or leave these meta roles. Users in these roles are trusted with management responsibilities of the assigned entities. 


\section{Overview of the Collaboration Specification Model}

In this section, we present the seminal elements of the collaboration specifi cation model that we developed in our project for supporting policy-driven construction of collaboration activities [23, 21]. In this overview, we focus on the following features of this model:

- Defi nition of an activity in terms of roles and shared objects.

- Nesting of activities, passing of objects to a nested activity, and assignment of users to roles in a nested activity.

- Event model for coordination of role operations.

- Defi nition of shared objects.

- Defi nition of a role and its operations.

\subsection{Activity Definition}

An activity defi nes a scope for shared objects, roles, and nested activities. We illustrate the basic elements of activity defi nition using an example of a course management related activities. We fi rst present the structure of an activity and its related concepts using a a schematic representation shown in Figure 1. Here a Course activity is defi ned with a nested activity named Examination. In an environment, there can be many instances of the Course activity, e.g. a chemistry course. The Course activity consists of an Instructor role, an Assistant role with possibly multiple teaching assistants as its members, and a Student role having all registered students as its participants. Participants in the Student role of the course are not allowed to join the Assistant role. In the nested Examination activity, the participant in the Examiner role creates an ExamPaper object. In this example, both the Instructor and the Assistant roles in the Course activities are made members of the Grader role of the Examination activity.

Each student in an Examination activity can instantiate a nested ExamSession activity. The ExamSession activity contains the roles Candidate and Checker. Only the student creating this activity is assigned to the Candidate role, and one of the participants in the Grader role is assigned to the Checker role. When an ExamSession activity instance is created, references to the ExamPaper object and a new AnswerBook object are passed to it. 


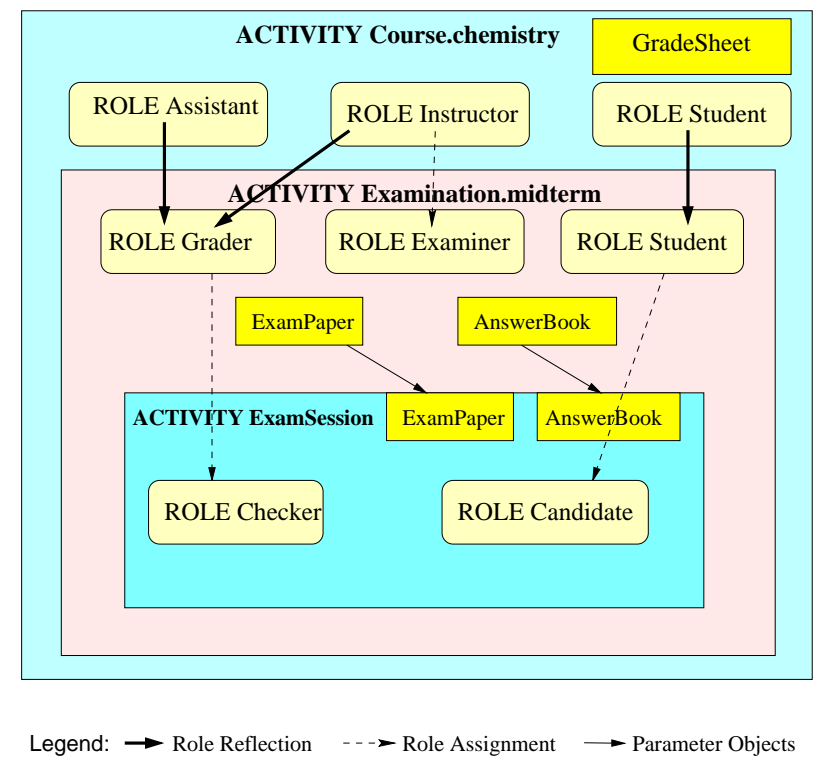

Figure 1: Hierarchical Structuring of Collaborative Activities

In the specifi cation model, an entity (such as activity, object, or role) encapsulated in the scope of an activity can be referenced by a fully qualifi ed name. Within an activity, one can refer to its current instance using the pseudo variable thisActivity, and its parent activity using parentActivity. The user executing an operation is identifi ed by thisUser. Within a role's operation, one can refer to the role by thisRole. The pseudo variable this refers to its immediate nesting entity, which can be an activity, role, object, or operation.

The basic schema for an activity specifi cation is shown below, where [ ] encloses optional terms.

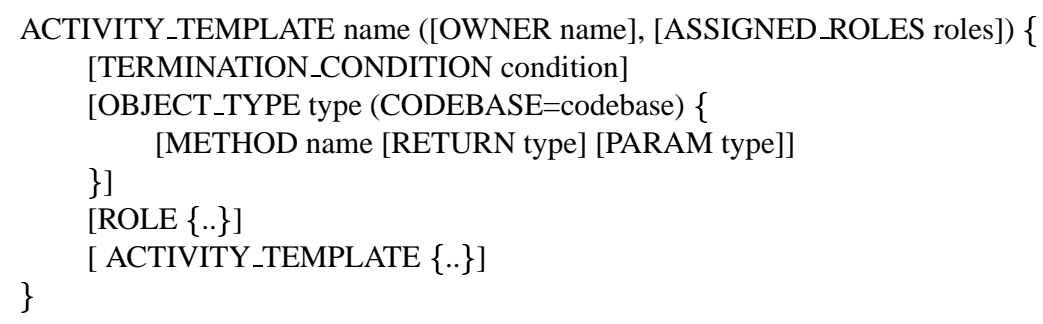

Figure 2, shows the complete specifi cation of the nested Examination activity of our course example. The various elements of this activity specifi cation are discussed in the following parts of this section. 


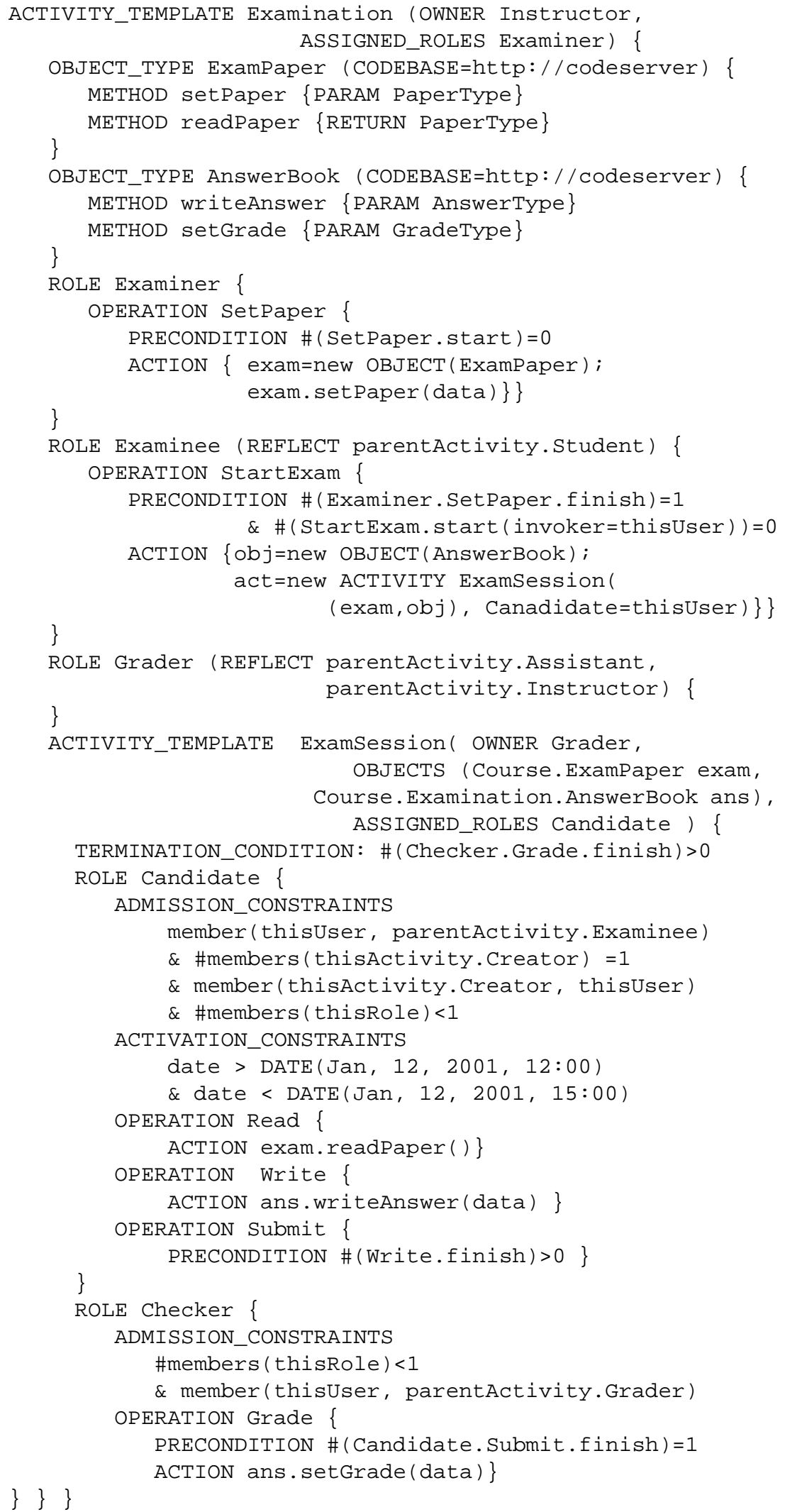

Figure 2: Specifi cation of Collaborative Examination Activity Template 


\subsection{Event Model}

Events and event counters are used in our model for specifying coordination and dynamic security policies. Event types are related to different kinds of entities such as activities, roles, operations, and objects. For example, instantiation of an activity, execution of a role operation, admission of user in a given role etc. represent different types of events. The multiple occurrences of a given event type — such as multiple executions of an operation — are represented by a list. We provide a count operator \# on lists. Hence, \#(eventName) returns the number of times the event has occurred.

We use event counters for synchronization specifi cation based on the model presented in [14]. Related to each role operation are two types of events: start, and finish. For example, role-name.op.start or role-name.op.finish. For a role, we also have event types defi ned for join, leave, admit, and remove operations. For each activity, we have start and finish events.

In our model, one can also specify a derived event type by fi ltering an event list based on its attributes. For example, for a role operation execution, we can defi ne a fi lter based on invoker id, such as opName. start (invoker=John) . We can count how many times a user has invoked an operation using \# (opName. start (invoker=John)) .

Events related to the physical environment can be imported from the underlying runtime system, if the specifi cation requires such events for context sensitive policies. For example, the collaboration system may need to detect the presence of a participant in a specifi c physical location to enforce context based access control.

\subsection{Shared Object Specification}

Shared objects are represented in our specifi cation model only in terms of their types and method signatures, keeping the semantics and implementation details transparent. A specifi cation for an object includes a type name to facilitate parameter bindings of operations in roles, a codebase to load the class of the object, and method signatures. The specification of an ExamPaper object type is shown in Figure 2. In addition of a codebase location, the object type has two method signatures defi ned: setPaper and readPaper. The specifi cation of the types of the parameters in these methods are not shown, which are mapped to some Java object types or primitive types using XML DTD.

Our system supports both RBAC as well as traditional DAC. We can specify access control at the granularity of the methods invoked on these objects. For an object, access control is derived from the various roles' operations involving that object. Based on the security and coordination requirements specifi ed in a collaboration, our system derives 
appropriate policy modules, which are used by the object servers to control access to their objects [23]. Moreover, after an object is created the owner can specify additional access control based on the traditional DAC models.

\subsection{Definition of Roles}

In our model, a role can be viewed as a protection domain with a set of privileges to perform certain tasks in the shared workspace defi ned by its activity's scope. A role defi nition involves specifi cation of two aspects:

- Meta-level policies in regard to admission of users to the role.

- Role related operations and associated preconditions for coordination and dynamic security requirements.

A role is defi ned in the scope of an activity and it can refer to the objects and other roles in that activity. It can invoke methods on the objects in that activity. As detailed below, a role specifi cation includes role owners, reflected roles, admission constraints, activation constraints, and role operations with their preconditions. The basic terms of a role specifi cation are shown below, where [ ] encloses optional terms.

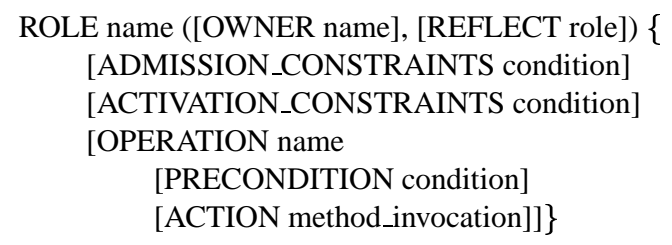

The owner of a role must be an existing role from its outer scope. If the owner is not specifi ed in a role defi nition, the owner of its activity is the owner. If the activity owner is not specifi ed, the default owner of any activity is the owner of its parent activity. For the top level activity, a meta-role Convener is defi ned as the owner. The owner of a role can admit users to it, subject to the specifi ed admission constraints; it can also remove an existing participant. Importantly, a role is managed by participants in its owner role. For example, in Figure 2, the Instructor role from the outer scope is specifi ed as the owner of the Examination activity. As no owner is specifi ed for the individual roles in this activity, by default, the Instructor becomes the owner of the Examiner, Examinee and Grader roles.

In the specifi cation model several functions are defi ned for a role. A boolean function member(user, role) checks if a participant is present in a role. The function members(role) gives the list of participants in a role. Hence, a count 
of the participants admitted in a role is given by \#(members(role)). A role defi nition may specify privacy constraints as to which other roles are permitted to query its participants' identities.

\subsection{Role Admission Constraints}

These constraints control a user's admission to the role to meet the security requirements stated in Section 3 . For example, the Assistant role in Figure 1 can have several admission constraints, as shown below.

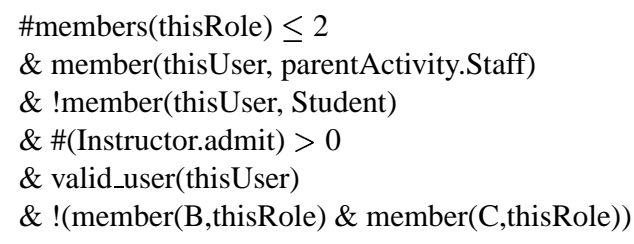

This example illustrates the following aspects of security requirements:

- Static separation of duties constraint specifying that a student in the Course activity cannot join the teaching assistant role.

- Role cardinality constraint requires that the member count for this role cannot exceed two.

- Previous role membership constraint, which requires that the person joining this role must already be a member of the Staff role defi ned in the parent activity.

- A user is admitted to this role only after some user has been assigned to the Instructor role.

- A "user-user separation of duties" requirement specifying that users $B$ and $C$ both cannot be assigned concurrently to this role.

\subsection{Role Reflection and User Assignment}

A nested activity may need to have access to the objects in the scope of its parent activity, or a role in the parent activity may need to be bound to a role in a nested activity. For this purpose, an activity defi nition needs mechanisms for role binding and passing object references as parameters to an activity instance. A role in the parent activity can be bound to a role in the child activity in the static defi nition. We refer to it as role reflection, which means that all the 
members of the parent role implicitly become members of the role in the child activity. Removal of a participant from the reflected role, also implies removal from the role in the child activity. A participant in the reflected roles (i.e. a role in the parent activity) gains expanded privileges comprising of the operations of the child activity role. Moreover, the child activity role can have operations that access the objects in the scope of the reflected role. However, a participant of the reflected role has to comply with the child role's admission constraints if any. In our model, roles are not defi ned based on permission inheritance [15, 13], rather defi ned in the scope of hierarchically nested activities. In Figure 2, the Student role is reflected in the Examinee role of any of the nested Examination activities.

The specifi cation also indicate assignment of users to certain roles at the time of activity creation. For example, in Figure 2, users must be assigned to the Examiner role in the Examination activity, and the Candidate role in the ExamSession activity. The user assignments maybe specifi ed as part of the activity creation specifi cation. In Figure 2, the examinee invoking the StartExam operation is assigned to the Candidate role of the new ExamSession activity instance. The users specifi ed to be assigned to a role also need to comply with the role's admission constraints. As opposed to role reflection and role assignment, when a user attempts to join a role directly, absence of a previous qualification constraint in the admission constraints prevents the user from joining.

\subsection{Operation Specification}

An operation specifi cation includes a name, and may include a precondition and an action. The precondition must be true when the operation is invoked. The preconditions associated with operations allows one to specify coordination constraints as well as various dynamic security requirements, such as condition-based access control, dynamic "separation of duties", context-based access control.

The action part of an operation may invoke an object method, or create a new object or a nested activity. If the action part is empty, then the operation is used primarily for coordination purposes. A keyword new is reserved for specifying creation of an object or activity. Follows an example of an operation specification of the Examiner role in the Examination activity as shown in Figure 1. The operation SetPaper can be performed only once as specifi ed by the precondition. This operation results in creation of an object exam of type ExamPaper and an invocation of the setPaper method of this object.

Use of preconditions enables us to specify fi ne grain "separation of duties" policies, like the "object based sepa- 


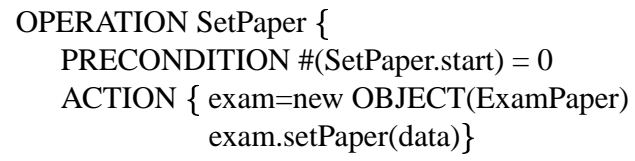

ration of duties" and the "operational separation of duties". For example, in an offi ce system, a manager may prepare an invoice and approve an invoice, but should not be able to approve his/her own invoice. This specifi cation is shown below which can be also considered as an example of an "operational separation of duties" policy.

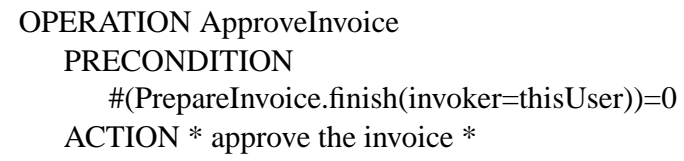

Preconditions also enable us to specify coordination constraints, for both inter-role and intra-role coordination. In Figure 2, a student in the Examinee role can not execute the StartExam operation until the Examiner has set the exam paper. The precondition for this operation also illustrates an intra-role coordination policy, which allows each participant in the Examinee role to independently start an exam session. This illustrates the independent participation model for the members in the Examinee role. The precondition of the SetPaper operation in the Examiner role illustrates the cooperative participation model for the members in this role - only one member in this role can execute the SetPaper operation.

\subsection{Role Activation Constraints}

Role activation constraints must be true when a user executes a role operation. In contrast, role admission constraints are checked only when a user is admitted to a role. Such constraints may not be valid when an operation is executed. Role activation constraints apply to all role operations and can be viewed as preconditions that are common to all operations of a role. Certain security requirements can be enforced only by activation constraints. A "dynamic separation of duties" constraint, such as a user should not activate two roles at the same time needs to be specifi ed as part of role activation constraints. Similarly, previous qualifications that must be ensured during role operation invocation need to be specifi ed as activation constraints. For example, the following activation constraint of the 
Assistant role in Figure 1 ensures that the role can perform any operation only as long as the participant is a member of the Staff role. Moreover, minimum cardinality constraints, which specify a minimum number of participants that

ACTIVATION_CONSTRAINTS

member(thisUser, parentActivity.Staff)

must be present before any role operation can be performed, are specifi ed as activation constraints.

In the following example, we present several activation constraints for an admission committee member role of a computer engineering department. Such a role may specify a minimum (e.g. 2) number of participants to be present for the committee to be active. Moreover, a constraint can be that at least a member from both the computer science and the electrical engineering departments must be present during role activities.

\#members(thisRole) $>2$

$\& \#$ (members(thisRole) $\cap$ members(EE.Professor) $)>0$

$\& \#($ members (thisRole $) \cap$ members(CS.Professor $))>0$

\section{Policy Based Construction of Collaboration Environment}

A collaboration environment in our model is realized in several steps through a policy driven middleware that we have developed [23]. Initially, the coordination and security policy for a collaboration is specifi ed based on the schema. From the specifi cation, various policy modules are derived for different kinds of requirements: role based security, object level access control, and secure event notifi cation for coordination. A generic middleware facility discussed in [23] provides a set of generic components which are coupled with these application specifi c policy modules to realize the desired runtime collaboration environment.

In our distributed execution model [22], entities - roles, objects, and activities - are managed in a decentralized manner. In our collaboration model, all users are not equally trusted. To ensure the proper enforcement of various collaboration policies, the collaboration entities need to be maintained at trusted sites. We derive a distributed trust relationship among the owners of various entities based on owner assignment as specifi ed in the activity defi nition. As roles from the outer scope of an entity are specifi ed (or assigned through default rules as discussed in Section 4) as the owner of that entity, the owners represent a hierarchical trust relationship. An entity is managed at its owner's site. 
In our specifi cation model, the coordination policies are specifi ed based on serialized evaluation of dependent operation preconditions. In a decentralized environment, where users' actions are communicated by means of events, communication of coordination states to other users may be delayed. In the absence of a central coordinator, such delays may cause coordination inconsistencies. In [22], we have presented a technique for for ensuring coordination consistency among distributed entities.

Coordination and dynamic access control are based on events communicated among distributed entities. To ensure integrity of our collaboration system, the middleware needs to ensure reliable delivery of events, and that events are not falsifi ed or omitted. In [22], we present a protocol which ensures integrity of events by subscribing corroborating events.

\section{Related Work}

Our goal is similar to those of COCA [12] and DCWPL [2] in their approach of constructing a distributed collaboration environment from a high level specifi cation. COCA [12] is a logic-based coordination policy specifi cation language for interactive CSCW applications which views security policy as an integral part of coordination policy. At the implementation level, COCA is tied with IP multicast models and Prolog. Recent work on COCA [7] applied formal verifi cation techniques for ensuring privacy in user presence awareness systems. DCWPL [2] addresses user level mechanisms to deal with group interaction issues and is limited to its predefi ned policies and functions. The specifi cation of confi dentiality using $\mathrm{Z}$ notation in [6] is limited to its theoretical foundation and lacks an implementation model.

The concept of role has been used in many CSCW systems, e.g. XCP [18], MPCAL [8], Quilt [11]. These applications use roles to represent groups of users with different tasks within a collaboration. They do not satisfy the access control requirements specifi ed in this paper. Decentralized management of role memberships based on role certifi cates in a distributed service model is presented in [9]. Suite [4] presents an access-control model for multiuser GUI interfaces, mainly for coordination of shared editing-based synchronous collaboration. For that, it deals with fi ne grain access rights on shared data. In contrast, our work focuses on a broad range of application wide security requirements, addressing the needs of data confi dentiality, integrity, and dynamic security policies. Intermezzo [5] is 
one of the fi rst systems to introduce the basic concepts of role based policies, with primary focus on user-presence awareness environments.

A task-based constraint specifi cation language for workflow management systems is discussed in [1]. There, constraints are specifi ed with a mapping between roles and tasks. In contrast, our work specifi es both the security and the coordination policies, with realization of such policies in an implemented system. SecureFlow [10] imposes workflow authorization constraints on tasks using Authorization Template (AT), which is a tuple specifying privileges to be granted to a subject of a given role on an object of a given type during a specifi ed time interval. There, the permissions are activated based on tasks. In contrast, an activity in our model is a higher level abstraction than AT, representing a collaboration pattern, which involves multiple roles, objects, and their coordination. An activity specifi cation may contain multiple tasks or operations and is able to capture workflow stages. Moreover, in [10] roles are bound to tasks at runtime based on AT. This requires certain rules to resolve conflicts when multiple roles for a user can be bound to a task. This approach is mainly to impose role based access control on existing workflow tasks. In our specifi cation, role and activity modeling incorporates collaboration tasks, and tasks are encapsulated as operations within the role defi nition. This provides a deterministic view of roles privileges.

\section{Conclusion}

We have presented in this paper a role-based specifi cation model for collaboration systems based on requirements of dynamic security policies, role admission and activation constraints, and separation of duties constraints. The specifi cation model unifi es coordination specifi cations with dynamic access control policy specifi cations. Roles are defi ned and instantiated in the context of activities. We have presented the concept of activity template, which enables dynamic creation of activities and hierarchical structuring of sub-activities. An activity defi nes a protection domain for roles, objects, and operations. We have implemented this specifi cation model using an XML schema and shown how the model supports different coordination and security requirements characteristic of collaboration systems. Policy modules are derived from the XML specifications and are integrated with a generic middleware for the automated realization of collaboration environments. This approach represents a novel technique for constructing the runtime environments for collaboration systems from their high level specifi cations. 


\section{References}

[1] E. Bertino, E. Ferrari, and V. Atluri. A Flexible Model Supporting the Specifi cation and Enforcement of Rolebased Authorizations in Workflow Management Systems. In ACM Workshop on Role-based Access Control, pages 1-12, 1997.

[2] M. Corts and P. Mishra. DCWPL: a programming language for describing collaborative work. In Proc. of CSCW'96, pages 21 -29, November 1996.

[3] S. Demurjian, T. Ting, and B. Thuraisingham. User-role based security for collaborative computing environments. Multimedia Review, 4(2):40-47, Summer 1993.

[4] P. Dewan and H. Shen. Controlling access in multiuser interfaces. ACM Transaction Computer-Human Interaction, 5(1):34 - 62, March 1998.

[5] W. K. Edwards. Policies and Roles in Collaborative Applications. In Proc. of CSCW'96, pages 11-20, 1996.

[6] S. Foley and J. Jacob. Specifying Security for Computer Supported Collaborative Computing. Journal of Computer Security, 3(4):233-253, 1995.

[7] P. Godefroid, J. D. Herbsleb, L. J. Jagadeesany, and D. Li. Ensuring Privacy in Presence Awareness Systems: An Automated Verifi cation Approach. In Proc. of CSCW'2000, pages 59-68, December 2000.

[8] I. Greif and S. Sarin. Data sharing in group work. ACM Transactions on Information Systems, 5(2):187-211, 1987.

[9] R. Hayton, J. Bacon, and K. Moody. Access control in an open distributed environment. In IEEE Symposium on Security and Privacy, pages 3-14, 1998.

[10] W.-K. Huang and V. Atluri. SecureFlow: a secure Web-enabled workflow management system. In ACM Workshop on Role-based Access Control, pages 83 - 94, 1999.

[11] M. Leland, R. Fish, and R. Kraut. Collaborative Document Production using Quilt. In Proc. of CSCW'88, pages 206-215, 1988. 
[12] D. Li and R. Muntz. COCA: Collaborative Objects Coordination Architecture. In Proc. of CSCW'98, pages $179-188,1998$.

[13] E. C. Lupu and M. Sloman. Reconciling Role-Based Management and Role-Based Access Control. In ACM workshop on Role-based Access Control, pages 135-141, 1997.

[14] P. Roberts and J.-P. Verjus. Towards Autonomous Descriptions of Synchronization Modules. In Proc. of IFIP Congress, pages 981-986, 1977.

[15] R. Sandhu, E. Coyne, H. Feinstein, and C. Youman. Role-Based Access Control Models. IEEE Computer, 29(2):38-47, February 1996.

[16] R. S. Sandhu. Transaction control expressions for separation of duties. In Fourth Annual Computer Security Application Conference, pages 282-286, December 1988.

[17] R. Simon and M. Zurko. Separation of duty in role-based environments. In 10th Computer Security Foundations Workshop, pages $183-194,1997$.

[18] S. Sluizer and P. M. Cashman. XCP: an experimental tool for managing cooperative activity. In Proceedings of the 1985 ACM Thirteenth Annual Conference on Computer Science, pages 251 - 258, 1985.

[19] R. K. Thomas and R. S. Sandhu. Conceptual Foundations for a Model of Task-based Authorizations. In In Proceedings of IEEE Computer Security Foundations Workshop , pages 66-79, 1994.

[20] J. E. Tidswell and T. Jaeger. Integrated constraints and inheritance in DTAC. In ACM Workshop on Role-based Access Control, pages 93 - 102, July 2000.

[21] A. Tripathi. Adaptive middleware: Challenges designing next-generation middleware systems . Communications of the ACM, 45(6):39-42, June 2002.

[22] A. Tripathi, T. Ahmed, and R. Kumar. Secure Management of Distributed Collaboration Systems. Technical report, Dept. of Computer Science, Univ. of Minnesota, Aug. 2002. Available at http://www.cs.umn.edu/Ajanta.

[23] A. Tripathi, T. Ahmed, R. Kumar, and S. Jaman. Design of a Policy-Driven Middleware for Secure Distributed Collaboration. In Proc. of ICDCS'2002, pages 393 - 400, July 2002. 